\title{
Otimização Multicritério dos Problemas de Ângulos de Feixe e Mapa de Fluência para Tratamentos Radioterápicos IMRT
}

\author{
Thiago S. Marques ${ }^{1}$, Elizabeth F. G. Goldbarg ${ }^{1}$, Sílvia M. D. M. Maia ${ }^{1}$ \\ ${ }^{1}$ Departamento de Informática e Matemática Aplicada \\ Universidade Federal do Rio Grande do Norte (UFRRN) - Natal - RN - Brasil \\ thiago@ppgsc.ufrn.br, \{beth,silvia\}@dimap.ufrn.br
}

\begin{abstract}
Cancer is a disease that affects thousands of people worldwide, and each year the number of people diagnosed with some type of cancer grows. The treatment of cancer based on radiotherapy is the object of study in this paper. This work addresses two computational problems involved in the planning stage of radiotherapy treatments: the selection of the angles of the radiation beams and the determination of the amount of radiation that each beam should emit, also known as Beam Angle Optimization and Fluency Map Optimization. Mono-objective and multiobjective algorithms with mathematical programming models were developed to solve problems. The implemented algorithms were tested with a set of real cases of liver and prostate cancer and their performances are reported in terms of the computational time spent and the quality of the solutions.
\end{abstract}

Resumo. O câncer é uma doença que acomete milhares de pessoas no mundo inteiro, e a cada ano o número de pessoas diagnosticadas com algum tipo de câncer cresce. $O$ tratamento de câncer baseado em radioterapia é o objeto de estudo deste trabalho. Este trabalho aborda dois problemas computacionais envolvidos na etapa de planejamento dos tratamentos radioterápicos: a seleção dos ângulos dos feixes de radiação e a determinação da quantidade de radiação que cada feixe deverá emitir, também conhecidos como Otimização do Ângulo de Feixe e Otimização do Mapa de Fluências. Algoritmos mono-objetivo e multiobjetivo com modelos de programação matemática foram desenvolvidos para resolução dos problemas. Os algoritmos implementados foram testados em um conjunto de casos reais de câncer de fígado e próstata e seus desempenhos são reportados no que diz respeito ao tempo computacional dispendido e qualidade das soluções encontradas.

\section{Introdução}

O número de pessoas diagnosticadas com algum tipo de câncer, no Brasil e no mundo, cresce a cada ano. Segundo a Organização Mundial da Saúde (OMS) estima-se que haverão 20 milhões de casos novos até 2025. Um estudo realizado em 2012 pela International Agency for Research on Cancer (Iarc), intitulado de projeto Globocan, apontou que mais de $60 \%$ dos casos novos de câncer ocorreram em países em desenvolvimento. Um índice ainda mais alarmante diz respeito a taxa de mortalidade para esses casos, onde $70 \%$ ocorreram nesses mesmos países. De acordo com o Instituto Nacional de Câncer José Alencar Gomes da Silva (INCA) no ano de 2016 estimou-se 61.200 casos novos de câncer de próstata e 57.960 casos novos de câncer de mama, no Brasil. 
Pesquisas com intuito de avançar tecnologicamente os possíveis tratamentos, dos mais variados tipos de câncer, são encorajadas no meio cientifico. O planejamento de tratamentos baseados em terapia com Radiação de Intensidade Modulada (IMRT, do inglês Intensity Modulated Radiation Therapy), no que remete-se a otimização, é dividido em três tarefas [Ehrgott et al. 2008]: problema da otimização dos ângulos dos feixes, problema da otimização da matriz de intensidade, e por último, o sequenciamento do colimador multilâminas. O objeto de estudo desse trabalho concentra-se nos dois primeiros problemas. Sendo assim, esse trabalho tem por objetivo estudar o Problema da Otimização dos Ângulos do Feixe (OAF) e o Problema da Otimização do Mapa de Fluências (OMF).

\section{Definição dos problemas}

Consideramos que o corpo do paciente é discretizado, de modo que cada órgão é dividido em pequenos volumes chamados voxels. Os feixes de radiação são discretizados em subfeixes, isto é, frações de um feixe.

A forma geral do problema OAF pode ser expressa pela formulação (1)-(2), em que $\mathcal{K}$ é um conjunto de ângulos, $N$ é um inteiro e $f$ é uma função que mapeia $\mathcal{C} \subseteq \mathcal{K}$ para um número real [Cabrera et al. 2016]. O conjunto $\mathcal{C}$ é chamado configuração do ângulo de feixe $(\mathrm{CAF})$. $\mathrm{O}$ problema do $\mathrm{OAF}$ visa encontrar um $\mathrm{CAF} \mathcal{C}$ que minimize alguma função $f$.

Minimize $f(\mathcal{C})$

Sujeito à:

$$
\mathcal{C} \subseteq \mathcal{K},|\mathcal{C}|=N
$$

A função $f: \mathcal{C} \rightarrow \mathbb{R}$ geralmente mede alguma propriedade da dose que irradia um órgão ou tumor. Essa formulação admite que a função $f$ seja substituída por um conjunto de funções, caracterizando um problema multiobjetivo. A equação (3) expressa a dose depositada em uma estrutura (órgão), onde $A_{r}$ é a matriz de deposição de doses que descreve a taxa na qual a radiação é depositada nos voxels do órgão $r$, e $x$ denota o mapa de fluência, que descreve o tempo de exposição à radiação.

$$
d_{r}=A_{r} * x
$$

O problema do OMF pode ser definido pelas expressões (4)-(5), em que a função objetivo $g$ modela alguma característica da dose fornecida pelo mapa de fluência $x \in \mathbb{R}^{n}$. O conjunto de mapas de fluência que contém apenas feixes de $\mathcal{C}$ é indicado por $X(\mathcal{C})$.

$$
\begin{aligned}
& \text { Minimize } g(x) \\
& \text { Sujeito à: } \\
& \qquad x \in X(\mathcal{C})
\end{aligned}
$$

Podemos perceber que enquanto a função (s) $f$ mapeia um conjunto de ângulos em um valor real (ou vetor de valores na formulação multiobjetivo), a função $g$ mapeia o mapa de fluência $x$ em um valor (ou vetor) real. 
A Figura 1 [Breedveld et al. 2018] exibe a visão geral dos problemas descritos. No canto superior direito da imagem, a fonte de radiação gera um feixe ionizante que é disparado contra o colimador multi-folhas (MLC). O MLC permite modelar a forma do feixe de radiação, sendo este discretizado em sub-feixes. Quanto mais tempo um sub-feixe permanece "aberto", maior é a intensidade desse sub-feixe, e por consequência maior é a dose resultante no paciente. Na parte inferior da figura é apresentada o comportamento de um feixe de radiação baseado em fótons, onde a cor vermelha indica uma dose alta e a azul uma dose baixa. A radiação é lançada da direita para a esquerda a partir de um único sub-feixe aberto. A largura desse sub-feixe é indicada pelo tamanho do quadrado da grade. Devido ao efeito de dispersão de partículas, a dose do feixe de radiação é mais larga que a abertura do feixe. Como consequência, tecidos que circundam a área irradiada também sofrem dano da radiação.
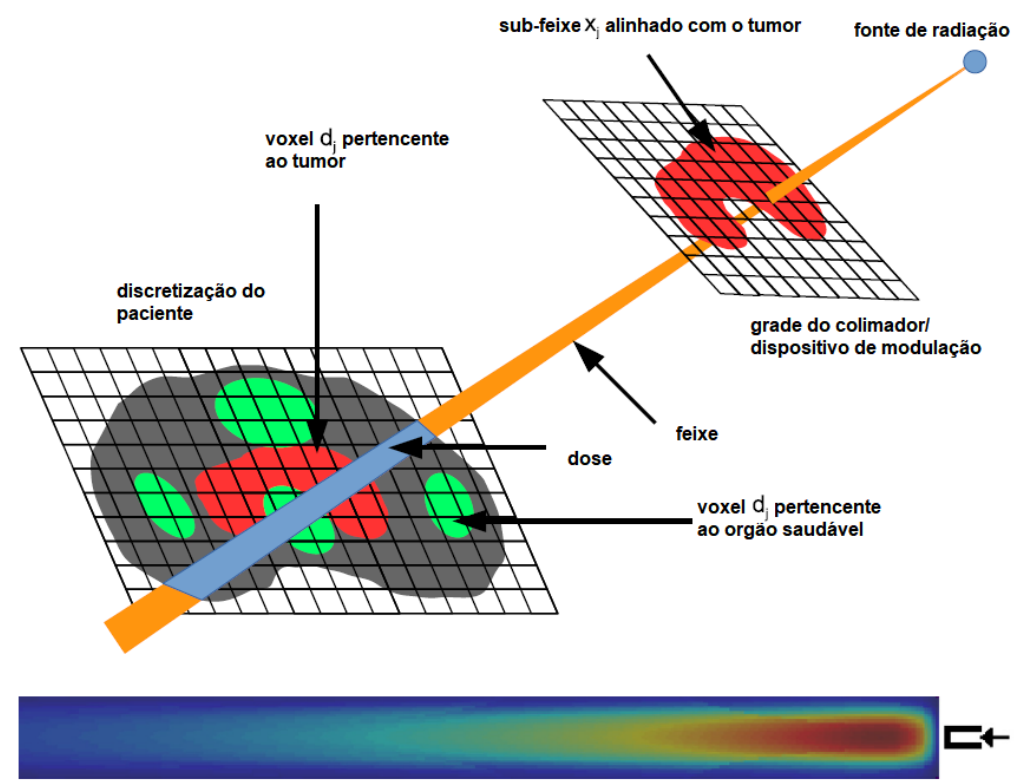

Figura 1. Representação dos problemas [Breedveld et al. 2018].

\section{Contribuições}

Foram propostos 4 modelos de programação matemática para o problema OMF, e um modelo multiobjetivo para o mesmo. Entre os 4 modelos propostos, 3 são de programação linear e o outro de programação quadrática. Foi apresentado ainda um estudo de desempenho dos modelos propostos em um conjunto de 20 instâncias reais, sendo 10 instâncias de câncer de fígado e 10 instâncias de câncer de próstata. Esses modelos foram desenvolvidos visando características especificas do problema, assim o trabalho contribui oferendo opções de modelos que atendam as necessidades especificas de cada caso. Como quando, por exemplo, o tumor está próximo a um órgão que não deve receber um grande quantidade de radiação. O modelo LP3 pode ser o mais adequado, uma vez que ele procura minimizar a dose máxima nesse órgão que circunda o tumor.

Foram apresentados 2 procedimentos de seleção de feixes, sendo o primeiro um algoritmo heurístico denominado Select $\mathcal{H}$ e o outro uma adaptação do modelo cônico desenvolvido por [Adler and Zibulevsky 2009]. A contribuição do algoritmo Select $\mathcal{H}$ é o 
fato de que a seleção de feixes acontece permitindo o uso de diferentes modelos de forma automatizada, explorando as melhoras características de cada modelo. Já a contribuição da adaptação do modelo cônico é percebida pela garantia de viabilidade do modelo, o que não acontecia no modelo original.

Ambos os algoritmos foram testados nos mesmos casos de teste já citados. Além disso, um algoritmo de otimização multi-critério foi desenvolvido para seleção de feixes e solução do mapa de fluência. A principal contribuição nesse algoritmo multi-critério foi a adaptação do método $\epsilon$-restrito, que agora possui uma quantidade de iterações limitada polinomialmente no número de objetivos.

\subsection{Modelos}

Cada modelo desenvolvido para o OMF se concentra em uma característica específica do problema. Elas são direcionadas a restringir doses em estruturas saudáveis e entregar a dose prescrita ao tumor, tanto quanto possível. A Tabela 1 resume a abordagem realizada por cada modelo. O símbolo $\checkmark$ nas colunas "Tumor" e "OAR" indica que há uma penalidade na função objetivo referente a essa estrutura. A característica de viabilidade indica que o modelo sempre oferece uma solução viável em relação às restrições matemáticas. Essa solução, no entanto, pode não atender todas as restrições demandadas pelo médico.

Em alguns casos, os modelos lineares podem não conseguir expressar algumas propriedades e relações entre os órgãos de risco, o tumor e as doses, então é necessário introduzir expressões não lineares. No entanto, modelos não lineares adicionam alguma dificuldade em relação aos métodos de solução. Nesses casos, há um balanço entre precisão da dose, expressividade do modelo e desempenho do método de solução. O símbolo $\checkmark$ na coluna "Não linearidade" indica que o modelo tem uma função objetivo não linear. O modelo QP1 introduz penalidades por desvios de dose quadráticos. A última característica, homogeneidade, indica que o modelo procura encontrar uma solução que distribua doses o mais homogêneas possível, em uma estrutura específica. O modelo LP2 possui essa propriedade.

\begin{tabular}{|l|c|c|c|c|c|}
\hline & Tumor & OAR & Viabilidade & Não Linearidade & Homogeneidade \\
\hline LP1 & $\checkmark$ & $\checkmark$ & $\checkmark$ & & $\checkmark$ \\
LP2 & $\checkmark$ & & & & \\
LP3 & & $\checkmark$ & & & \\
QP1 & $\checkmark$ & $\checkmark$ & & $\checkmark$ & \\
\hline
\end{tabular}

Tabela 1. Propriedades dos modelos propostos.

Uma inovação introduzida nos modelos projetados foi usar explicitamente as restrições de cálculo de dose nos modelos desenvolvidos, uma vez que isso proporcionou uma melhoria significativa no tempo necessário para resolver o problema. A seguir são exibidas as funções objetivo de cada modelo.

- LP1:

$$
\operatorname{Min} \sum_{i=1}^{m^{t}} \theta_{i}+\sum_{r \in R \backslash\{t\}} \sum_{i=1}^{m^{r}} \delta_{i}^{r}
$$


- LP2:

$$
\operatorname{Min} \sum_{i=1}^{m^{t}}\left(d_{i}^{t}-L B^{t}\right)
$$

- LP3:

$$
\operatorname{Min} \sum_{r \in R \backslash\{t\}} \max \left(d^{r}\right)
$$

- QP1:

$$
\operatorname{Min} \sum_{i=1}^{m^{t}}\left(d_{i}^{t}-L B^{t}\right)^{2}+\sum_{r=1}^{p}\left(\sum_{i=1}^{m^{r}}\left(d_{i}^{r}\right)^{2}\right)
$$

\subsection{Algoritmos}

O primeiro algoritmo desenvolvido foi o Select $\mathcal{H}$. A ideia geral do algoritmo é partir de um conjunto de ângulos vazio e iterativamente selecionar o ângulo mais promissor (menor valor objetivo) até atingir o número de ângulos desejado. Como em geral, planos com poucos ângulos $(<4)$ tendem a inviabilizar o modelo LP3, o algoritmo utiliza o modelo LP1 nas primeiras iterações para escolher o ângulo mais promissor e nas demais iterações utiliza-se o modelo LP3. Se em iterações seguintes o modelo LP3 falhar por duas vezes seguidas, usa-se para o modelo LP1 para escolher o ângulo naquela determinada iteração.

O segundo algoritmo implementado foi o algoritmo de duas fases, sendo que este resolve ambos os problemas OAF e OMF de maneira multiobjetivo. A primeira fase do algoritmo encontra um conjunto de feixes utilizando um dos algoritmos propostos para seleção de ângulos, ou seja, o Select $\mathcal{H}$ ou o modelo cônico. Em seguida, um algoritmo baseado no método do $\epsilon$-restrito é executado para gerar vários mapas de fluência ótimos, onde a otimalidade se refere a otimalidade de Pareto. Desse modo, um mapa de fluência é dito Pareto ótimo se for viável em relação a todas as restrições e nenhum objetivo puder ser melhorado sem necessariamente prejudicar pelo menos um outro objetivo.

\section{Resultados}

Para testar as potencialidades das abordagens propostas neste estudo, realizamos experimentos em um conjunto de instâncias disponibilizadas por [Breedveld and Heijmen 2017].

Foram testados 10 casos de câncer de fígado com 8 ou 9 órgãos em cada caso, além do tumor (PTV): estômago, medula espinhal, coração, esôfago, duodeno, rim direito (R), rim esquerdo (L) e pâncreas. Além disso foram testados 10 casos de câncer de próstata considerando as seguintes estruturas: reto, uretra, bexiga, pênis/bolsa testicular, cabeça femoral (D) e cabeça femoral (E).

O algoritmo de duas fases, encontrou uma média de 300 soluções não-dominadas, que podem ser avaliadas posteriormente a fim de definir a mais adequada para o paciente.

A Figura 2 apresenta os resultados para algumas instâncias na forma de diagramas de dose-volume. O diagrama mostra a dose de radiação no eixo horizontal e no eixo vertical mostra a porcentagem do volume de um órgão. Cada cor indica um órgão. Um ponto $(x, y)$ em uma curva representa que $x \%$ do volume do órgão correspondente recebe uma dose maior ou igual a y Gy. As curvas tem início no canto superior esquerdo e 


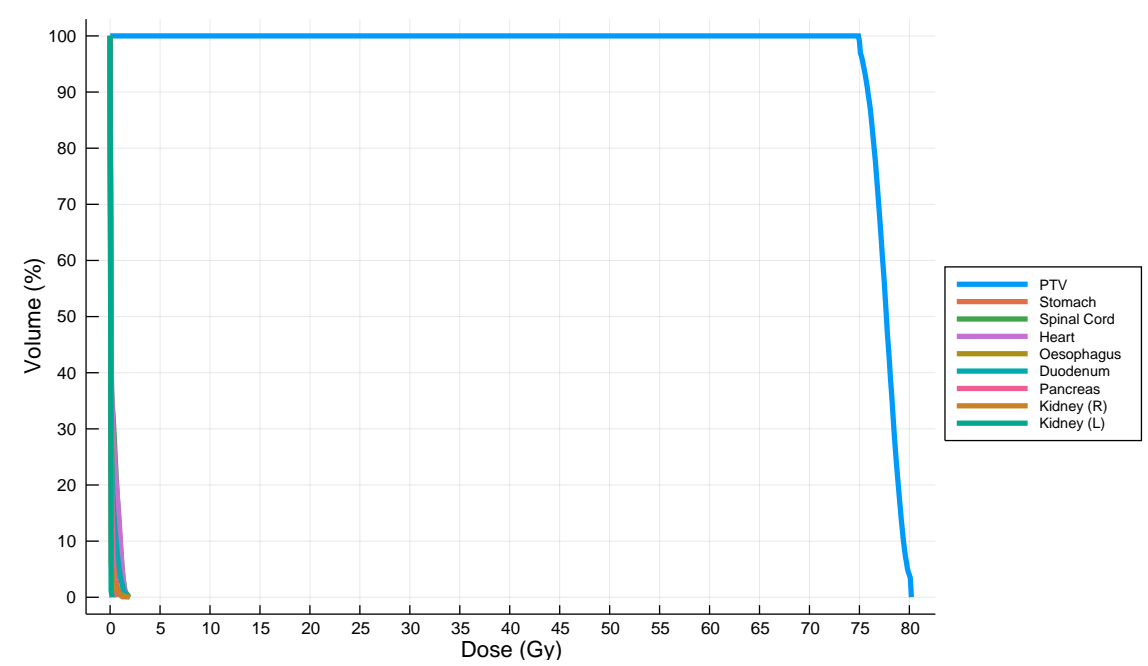

(a) Liver_02

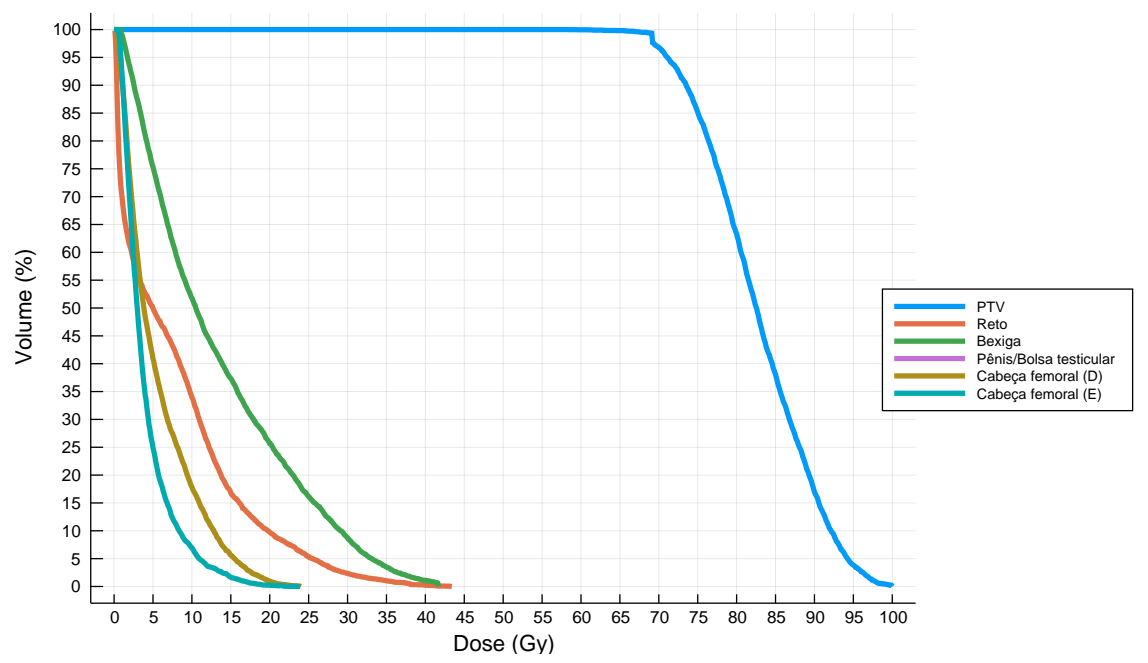

(b) Prostate_03

Figura 2. Histograma dose volume

terminam no canto inferior direito. Uma dose totalmente homogênea de $y$ Gy distribuída em uma estrutura é representada como uma linha horizontal que cai verticalmente em $y$ Gy em relação ao eixo da dose.

Na Figura 2a, referente a um caso de fígado, vemos que o PTV (tumor) recebe uma dose acima de 70 Gy em $100 \%$ do seu volume e nenhum órgão saudável recebe uma dose superior a $5 G y$, o que configura uma solução de alta qualidade, uma vez que preserva os órgãos do paciente o máximo possível. Já a Figura 2b, que mostra uma solução para um caso de próstata, apresenta mais dificuldades em manter doses mais baixas nos órgãos sadios, mais ainda assim consegue manter as prescrições médicas para a maioria dos órgãos, e também para o tumor.

\section{Conclusão}

Pelos resultados obtidos, pode-se perceber que as abordagens desenvolvidas apresentaram um bom desempenho para ambos os problemas, OAF e OMF, uma vez que soluções 
de alta qualidade foram encontradas pelas estratégias implementadas. No que diz respeito ao tempo computacional, os algoritmos executaram em 2,5 horas em média (com algumas exceções para as instâncias de fígado) e em 6 horas em média para as instâncias de próstata. Os casos de próstata mostraram-se mais desafiadores, uma vez que precisaram de um conjunto de feixes maior comparado as instâncias de fígado para serem resolvidos. O tempo de processamento levado pelos algoritmos para solucionar os problemas pode ser considerado clinicamente aceitável, ainda assim otimizações podem ser feitas a fim de melhorar o desempenho desses algoritmos.

\section{Referências}

Adler, A. and Zibulevsky, M. (2009). Sparse solution for the intensity-modulated radiotherapy problem using conic programming. Technical report, Computer Science Department, Technion.

Breedveld, S., Craft, D., Van Haveren, R., and Heijmen, B. (2018). Multi-criteria optimisation and decision-making in radiotherapy. European Journal of Operational Research.

Breedveld, S. and Heijmen, B. (2017). Data for trots-the radiotherapy optimisation test set. Data in brief, 12:143-149.

Cabrera, G., Ehrgott, M., Mason, A. J., Raith, A., et al. (2016). A matheuristic approach to solve the multiobjective beam angle optimization problem in intensity-modulated radiation therapy. International Transactions in Operational Research.

Ehrgott, M., Güler, Ç., Hamacher, H. W., and Shao, L. (2008). Mathematical optimization in intensity modulated radiation therapy. 4OR, 6(3):199-262. 